\title{
Frameless Fractionated Stereotactic Radiation Therapy
}

National Cancer Institute

\section{Source}

National Cancer Institute. Frameless Fractionated Stereotactic Radiation Therapy. NCI

Thesaurus. Code C128555.

A type of fractionated stereotactic radiotherapy that does not involve the use of a frame to hold the patient's head still or provide external reference points. 